\title{
Monitoramento da cadeia do frio: novas tecnologias e recentes avanços
}

\author{
Monitoring the cold chain: new technologies and recent advances
}

\author{
Wigberto Antonio Spagnol ${ }^{1 *}$, Vivaldo Silveira Junior', Ericsem Pereira', Nelson Guimarães Filho² \\ ${ }^{1}$ Universidade Estadual de Campinas (UNICAMP), Faculdade de Engenharia de Alimentos, Campinas/SP - Brasil \\ 2 Pleiades S.C.O. Digitais Ltda., Campinas/SP - Brasil
}

\section{*Corresponding Author}

Wigberto Antonio Spagnol, Universidade Estadual de Campinas (UNICAMP), Faculdade de Engenharia de Alimentos, Rua Monteiro Lobato, 80, Caixa Postal: 6121, CEP: 13083-862, Campinas/SP - Brasil, e-mail: waspagnol@gmail.com

Cite as: Monitoring the cold chain: new technologies and recent advances. Braz. J. Food Technol., v. 21, e2016069, 2018.

Received: June 23, 2016; Accepted: July 21, 2017

\section{Resumo}

A economia, atualmente, é impulsionada pela alta competitividade, exigindo que os setores de produção e logística sejam ágeis, flexíveis e com capacidade de ação para a solução de dificuldades. A logística é de extrema importância para as operações das cadeias de alimentos perecíveis, como carnes, peixes, lácteos, frutas e produtos hortícolas, assim como para determinados produtos da indústria farmacêutica, como vacinas, biomedicamentos e hemoderivados, que necessitam do uso da cadeia do frio devido à sua perecibilidade. A temperatura é o fator mais importante para a conservação da qualidade e manutenção da vida útil destes produtos, sendo a refrigeração um dos métodos mais amplamente utilizados para retardar o desenvolvimento de vários fatores que conduzem à sua deterioração. Dentre os desafios para a melhoria de incertezas da cadeia do frio, há a mitigação de riscos de ruptura da mesma. Sabe-se que há variações inevitáveis das condições de conservação dos produtos durante as etapas da cadeia de frio, as quais causam alteração da vida útil de alimentos perecíveis. Seja no transporte ou nas câmaras frias de armazenagem, há flutuação de temperatura em torno da condição ideal, sendo que ainda hoje, na maioria dos sistemas, o monitoramento da temperatura é realizado por pouquíssimos sensores. Embora poucas tecnologias de monitoração de temperatura sejam utilizadas para inibir os riscos de perda de qualidade e obter a segurança alimentar, estas são utilizadas de forma independente, nos diferentes elos da cadeia logística do frio. Esses desafios têm impulsionado esforços de grupos de pesquisas de países desenvolvidos para o aperfeiçoamento de aplicações de rede de sensores sem fio (RSSF) e de identificação por radiofrequência (RFID), com base em dispositivos sensores. Este trabalho aborda aspectos importantes da aplicação destas novas tecnologias, que são capazes de detectar a condição destes produtos perecíveis em tempo real, durante as operações logísticas, possibilitando evitar a perda, melhorar a qualidade dos produtos perecíveis e reduzir os custos.

Palavras-chave: Rede de sensores; RFID; Produtos perecíveis; Rastreabilidade; Segurança alimentar.

\section{Abstract}

The economy is currently driven by great competitiveness, demanding that the production and logistics sectors be agile, flexible and capable of action to solve difficulties. Logistics is of utmost importance to the operations of perishable food chains such as meats, fish, dairy, fruit and vegetable products, as well as for certain products in the pharmaceutical industry, such as vaccines, biomedicines and blood products that require the use of the cold chain due to their perishability. Temperature is the most important factor in the maintenance of quality and the shelf life of these products, cooling being one of the most widely used methods to slow the development of several factors that lead to product deterioration. Amongst the challenges for improving uncertainties related to the cold chain, is the mitigation of risks concerning its rupture. It is known there are inevitable variations in the storage conditions of the products during the stages of the cold chain, which cause changes in the shelf life of perishable foods. Both inside containers and in cold chambers there are temperature fluctuations around the ideal condition, and to this day, in most systems, monitoring of the temperature is measured by very few sensors. Although limited temperature monitoring technology is used to inhibit the risk of quality loss and improve food security, they are also used independently in the different logistics links of the cold chain. These challenges have driven efforts from some 
research groups in developed countries to improve the application of wireless sensor networks (WSN) and radio frequency identification (RFID), based on sensor devices. This paper addresses important aspects of the application of these new technologies that are able to detect the condition of these perishable products in real time during logistics operations, making it possible to avoid the loss of perishable products, improve their quality and reduce costs.

Keywords: Sensor network; RFID; Perishable products; Traceability; Food safety.

\section{Introdução}

O Brasil tornou-se um dos maiores produtores do mundo de carne de aves, de bovinos e de suínos, com estimativa de exportação de 6,25 milhões de toneladas dessas três commodities, em 2014 (ABPA, 2015). Mercadorias, como carnes congeladas, frutas e hortaliças das costas oeste e leste da América do Sul, representam um papel importante no suprimento mundial de alimentos perecíveis. De acordo com Jedermann et al. (2014c), o volume de exportações de alimentos perecíveis no mundo alcança um valor superior a 800 bilhões de dólares, sendo que as perdas são estimadas acima de $30 \%$.

Estudo de White (2007), relativo ao monitoramento de transporte refrigerado de alimentos perecíveis, demonstrou que ocorre um aumento da temperatura acima da temperatura especificada em $30 \%$ das viagens do produtor ao Centro de Distribuição (CD) e, em 15\%, nas viagens do CD para os locais de comercialização, no varejo.

De acordo com Brand (2014), na última década, houve uma mudança acentuada no transporte de produtos perecíveis, de transporte aéreo para transporte marítimo, em contêineres refrigerados.

Segundo Heidmann et al. (2013), o transporte em veículos refrigerados, tanto urbano como interurbano, pode constituir um ponto crítico na depreciação da qualidade do produto perecível, tendo em vista as variações de temperatura durante as operações de carregamento e descarga, ou mesmo durante todo o processo de transporte. Assim, de acordo com Tingman et al. (2010), cerca de um terço de alimentos frescos é descartado, devido às condições sanitárias e de conservação inadequadas, durante as etapas de distribuição.

A característica do consumo imediato de frutas e vegetais decorrente de sua perecibilidade, principalmente de hortaliças folhosas e frutas tropicais, além de exigir controles rígidos de temperatura, requer uma logística rápida e eficiente (RUIZ-GARCIA, 2008). Desta maneira, Mejjaouli e Babiceanu (2015) afirmam que as cadeias de suprimentos de alimentos precisam ser cada vez mais ágeis, para atender às necessidades de evolução dos mercados e atender a normas de segurança de qualidade, como a rastreabilidade.

Alguns dos desafios que surgem como decorrência inevitável dessa complexidade, são o monitoramento da vida útil e a mitigação de riscos de ruptura da cadeia do frio (LÜTJEN et al., 2013). Assim, o grande desafio é garantir uma "cadeia de frio" contínua, do produtor ao consumidor, reduzindo as perdas de qualidade dos produtos (CUIÑAS et al., 2014; RIZZO et al., 2011; RUIZ-GARCIA; LUNADEI, 2011).

Segundo Haan et al. (2013), esta situação é mais grave no caso de produtos farmacêuticos, uma vez que alterações inadequadas de temperatura, mesmo por curtos intervalos de tempo, podem inibir a eficácia do produto ou mesmo torná-lo perigoso.

A cadeia de frutas e hortaliças também apresenta uma grande complexidade, uma vez que os produtos possuem durabilidade diferentes, com diferentes exigências de temperatura, umidade etc., sendo necessário manter o frescor e a segurança dos alimentos em cada elo da cadeia logística, de modo a reduzir ao mínimo as perdas da qualidade, para satisfazer os clientes (AUNG; CHANG, 2014).

Para as frutas e hortaliças, a manutenção da temperatura adequada é o fator mais importante e mais simples, para diminuir os processos de metabolismo que permanecem após a sua colheita e que conduzem à rápida deterioração.

Em uma cadeia do frio, a vida útil, a qualidade e a segurança de frutas e hortaliças sofrem enorme influência dos fatores ambientais em todas as etapas da logística, especialmente da temperatura e da umidade relativa (AUNG; CHANG, 2014).

O processo de deterioração é geralmente proporcional à taxa de seu metabolismo, estando diretamente relacionado à temperatura. Com a redução do metabolismo, ocorre o retardamento de alterações indesejáveis, como amolecimento e mudanças na textura e na cor, além de perda de água, que também conduz à perda de peso. Além disso, a conservação, nas condições ideais de temperatura, é essencial e constitui uma maneira eficaz de retardar o crescimento de microrganismos deteriorantes (KADER, 2002).

De acordo com Ruiz-Garcia e Lunadei (2011), na logística de frutas e hortaliças, até 35\% da carga é perdida durante o transporte, sendo, a principal causa desta perda, as condições insuficientes de manutenção e controle de temperatura. Ben-Tzur et al. (2015) afirmam que, embora certo número de tecnologias seja utilizado para manter a qualidade de produtos perecíveis, estas são utilizadas de forma independente nos diferentes elos da cadeia logística, não ocorrendo uma interação e continuidade das informações entre esses elos. 
Assim, seria importante conhecer o histórico de temperatura do produto durante a sua cadeia logística; caso contrário, a vida útil é incerta (KETZENBERG; BLOEMHOF-RUWAARD, 2009). Segundo Ruiz-Garcia e Lunadei (2011), para diminuir os custos de logística de frutas e hortaliças, é essencial reduzir a quantidade de produtos que ficam expostos a condições inadequadas, para sua preservação durante o transporte e/ou entrepostos. Jedermann (2014) realçou a importância do monitoramento contínuo na cadeia de frutas e hortaliças, devido às variações inevitáveis durante as etapas da cadeia de frio, que, com certeza, causam a diminuição da vida útil.

De acordo com Ruiz-Garcia et al. (2009), o desenvolvimento de tecnologias de sensores sem fio entrou em uma nova fase, com os avanços decorrentes de sensores de detecção cada vez menores, das inovações da tecnologia de radiofrequência e de circuitos digitais. Tecnologia de sensores sem fio refere-se à Rede de Sensores Sem Fio (RSSF) e identificação por radiofrequência (RFID), com base em dispositivos sensores (JEDERMANN et al., 2014c). Ruiz-Garcia e Lunadei (2010) relatam que um benefício importante dos sistemas RFID com sensores e RSSF é a visibilidade das condições de conservação localizadas, que tais tecnologias podem proporcionar ao longo de uma cadeia de alimentos. A coleta de dados, inclusive em tempo real, das condições de conservação de frutas e hortaliças durante as diferentes fases, desde a colheita até o consumidor, possibilita melhorar as operações, enviando alertas decorrentes de condições inadequadas de conservação, proporcionando o registro automático de dados para conformidade, além da redução de custos de seguros. Lütjen et al. (2013) afirmam que o conceito de "contêiner inteligente", caracterizado pelo uso da tecnologia de rede de sensores no monitoramento mais preciso, e em tempo real, dos paletes de produtos em seu interior, representa uma nova forma de gestão no transporte de produtos perecíveis, a qual permite melhorar a sua eficiência, eliminando perdas.
Assim, os esforços de aperfeiçoamento do uso de tecnologia de sensores no monitoramento dos alimentos tende a crescer cada vez mais, como decorrência natural das exigências do mercado, para uma maior eficiência da cadeia logística.

\subsection{Tecnologia de RFID}

Os avanços atuais na tecnologia RFID com sensor, com recursos de data logger e com custos cada vez menores, têm proporcionado uma nova dimensão para a sua aplicação nas cadeias de alimentos, principalmente nas cadeias de alimentos perecíveis, cuja vida útil é curta (BADIA-MELIS et al., 2015). Além disso, a identificação automática da presença do produto e das condições de exposição, por meio de radiofrequência, permite maior confiabilidade e segurança da informação, e compartilhamento com todos os elos da cadeia de suprimentos (ZHANG; LI, 2012).

Segundo Zou et al. (2014), a tecnologia de RFID é considerada como a próxima tecnologia de impacto a ser implantada nas diversas cadeias de abastecimento.

Na Figura 1, está explicado o funcionamento de um sistema de monitoração com RFID, pelo envio da informação dos dados de um produto registrado na sua etiqueta. A informação nas etiquetas de RFID é captada por um conjunto de antenas e leitores, e interpretada pelo Middleware, por meio de radiofrequência, com a qual, ao contrário do código de barras, não há necessidade de se fazer escâner para cada embalagem próximo ao leitor ótico, evitando-se, também, o erro humano nessa ação (RUIZ-GARCIA, 2008; ZOU et al., 2014).

RFID é um chip com memória, um meio de armazenamento e recuperação de dados, que transmite informações por meio de ondas eletromagnéticas, usando um circuito integrado compatível.

Geralmente, as etiquetas RFID são classificadas em três categorias: passiva, semipassiva e ativa. As etiquetas
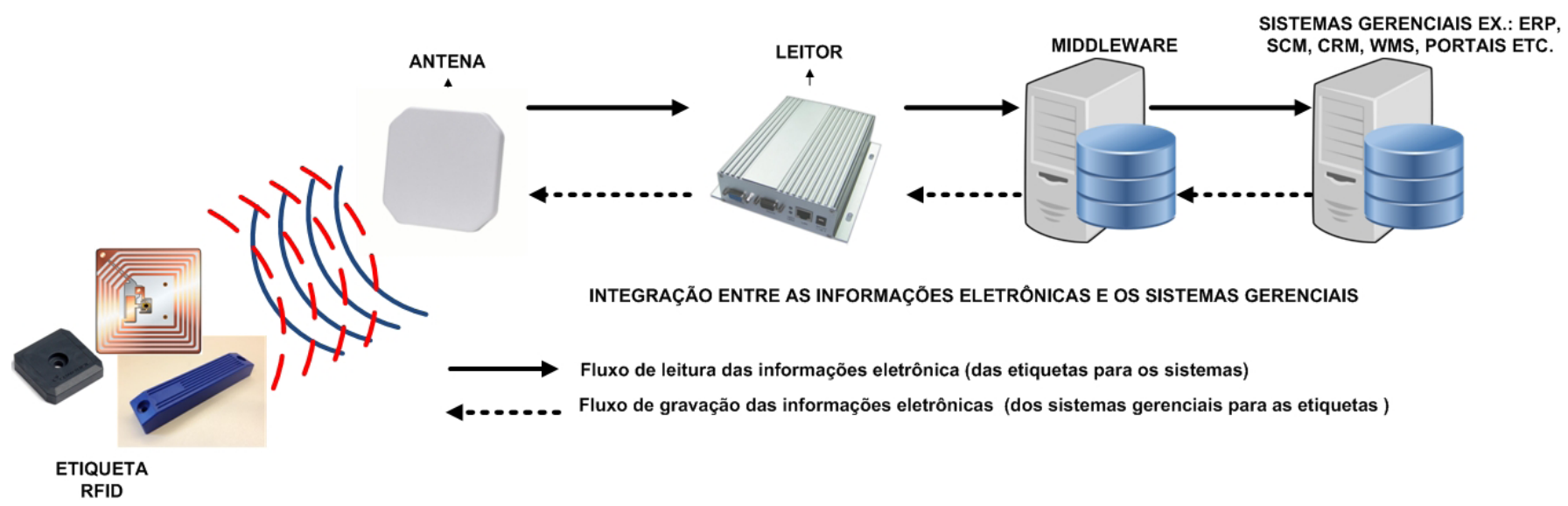

Figura 1. Funcionamento de um sistema de RFID (PEDROSO et al., 2009). 
passivas não têm qualquer fonte de energia, são ativadas por reflexão ou por modulação decorrente do campo eletromagnético emitido pelo leitor (RUIZ-GARCIA, 2008). As etiquetas passivas têm menor alcance de leitura (10 cm a 3 m), baixo custo e vida ilimitada (KUMARI et al., 2015). Estas etiquetas são aplicadas na maioria dos sistemas de identificação de produtos, devido ao seu baixo custo e dimensões reduzidas, uma vez que não requerem bateria. Etiquetas de RFID possuem frequências entre $125 \mathrm{kHz}$ (baixa frequência) e 13,6 MHz (alta frequência), sendo que $900 \mathrm{MHz}$ (UHF) são as mais comuns para sistemas passivos (ZOU et al., 2014).

As etiquetas ativas são alimentadas com uma bateria própria e, portanto, estão sempre ativas. Estas podem comunicar-se com o leitor em qualquer momento, possuem maior alcance de leitura, aproximadamente $100 \mathrm{~m}$, possuindo maior tamanho; seu custo situa-se entre 5 e 10 vezes acima do preço de etiquetas semipassivas (JEDERMANN et al., 2009).

Já as etiquetas semipassivas têm uma fonte de energia (bateria) para alimentar o sensor e armazenar os dados no chip. Ao contrário da etiqueta ativa, estas são ativadas pelo campo eletromagnético gerado pelo leitor para a comunicação. A bateria permanece inativa até ser energizada pelo campo gerado a partir de um leitor. Este mecanismo poupa bateria e aumenta a vida da etiqueta (KUMARI et al., 2015).

\subsection{Tecnologia de rede de sensores sem fio - RSSF}

A tecnologia de rede de sensores sem fio é uma evolução decorrente do avanço de tecnologias de medição, detecção, comunicação e de plataforma de dados em nuvem. A sua aplicação na cadeia do frio de produtos perecíveis possibilita o monitoramento de condições de conservação de alimentos que influenciam a sua vida útil, em toda a cadeia logística, proporcionando a tomada de decisões de forma mais eficiente (RUIZ-GARCIA et al., 2009). Neste contexto, a tecnologia de rede de sensores tem sido amplamente aplicada, como no desenvolvimento de contêineres inteligentes, nos quais sensores de temperatura, umidade relativa do ar e outros, distribuídos em todo o espaço interior, interagindo em rede, coletam as condições, transmitindo-as para um gateway (DITTMER et al., 2012; JEDERMANN et al., 2014a).

Assim, redes de sensores sem fio consistem de um grande número de dispositivos sensores (nós sensores ou, simplesmente, sensores) distribuídos em uma região de interesse. Estes sensores ativos têm conectividade por radiofrequência e podem estar conectados em rede, alimentados por baterias com comunicação e funções de computação limitadas, exigindo um algoritmo para realizar a sua auto-organização no ambiente monitorado (GAO, 2011). Cada nó pode ser equipado com diferentes sensores, tais como de temperatura, umidade, gases etc. (GARCIA et al., 2010).

De acordo com Kaushik e Singh (2013), há duas tecnologias de comunicação disponíveis para RSSF: ZigBee e Bluetooth, operando dentro de bandas industriais, científicas e médicas (ISM), com frequência de 2,4 GHz, que oferecem licença livre para operação, enorme reserva de espectro e compatibilidade em nível mundial. ZigBee é mais eficaz e econômica, em comparação com Bluetooth, com baixo consumo de energia. Em geral, com o aumento da frequência, aumenta-se a largura da banda, permitindo maiores taxas de dados, mas os requisitos de energia também são mais elevados e a distância de transmissão é consideravelmente mais curta (SHAN et al., 2004).

A maioria das RSSFs opera na faixa de frequência de 2,4 GHz (GAO, 2011). Esta faixa de frequência proporciona certas vantagens: uma alta largura de banda de $83 \mathrm{MHz}$, pequenas antenas e disponibilidade de vários sensores de rádio com base no padrão IEEE 802.15.4. Para aplicações ao ar livre e em edificações, uma potência de transmissão de $1,0 \mathrm{~mW}$ é suficiente para conseguir um intervalo de transmissão entre $10 \mathrm{~m} \mathrm{e}$ 100 m; no entanto, a propagação de sinal é amplamente impedida por alimentos que possuem altos teores de água (JEDERMANN et al., 2014c).

Bluetooth (IEEE 802.15.1) foi desenvolvido como protocolo sem fio para comunicação de curto alcance nas redes de área pessoal sem fio (PAN), em substituição aos cabos para dispositivos móveis. Bluetooth usa a frequência de 868 e $915 \mathrm{MHz}$, e as bandas de rádio de 2,4 GHz (RUIZ-GARCIA et al., 2009).

\section{Aplicações das tecnologias de monitoramento na cadeia do frio}

Fundamentado no conceito das tecnologias anteriormente apresentadas, foi feita uma revisão sobre aplicações de Tecnologias de Identificação por Radiofrequência e Tecnologias de Redes de Sensores Sem Fio, publicadas na última década.

De acordo com Ogasawara e Yamasaki (2006), o uso de etiquetas RFID com sensores de temperatura em aplicações de Análise de Perigos e Pontos Críticos de Controle (APPCC) na cadeia de frio, traz um gerenciamento eficaz do risco da temperatura, ao longo dos processos de transporte. Os avanços atuais na tecnologia RFID integrada a sensores têm proporcionado uma nova dimensão para a aplicação da tecnologia RFID nos sistemas de rastreabilidade de alimentos (BADIA-MELIS et al., 2015). Cuiñas et al. (2014) apresentaram estudos de projetos-piloto testando a capacidade da tecnologia de rede de sensores, para realizar a rastreabilidade em empresas de alimentos de vários setores: vinho, peixe e carne. Os autores concluíram que a utilização dessa tecnologia possibilita um fluxo constante de informação aos agentes de toda a cadeia, além de controlar 
os parâmetros críticos de conservação dos produtos e melhorar as informações de rastreabilidade da cadeia, estimando um retorno, em médio prazo, do investimento, entre quatro e cinco anos.

Lang et al. (2011) afirmam que a utilização de RSSF em contêineres refrigerados, associada ao modelo de estimativa da vida de prateleira de frutas, permite estabelecer uma nova forma de gestão na logística: FEFO dinâmico (First Expire First Out). Este modelo considera que o produto com menor vida de prateleira restante seja o primeiro a ser distribuído para a venda final.

Jedermann (2014) relata que o monitoramento da temperatura em tempo real, associado com modelos que estimam a vida útil, possibilita ações proativas pelos gestores que proporcionam redução de perdas financeiras na cadeia do frio de alimentos perecíveis. Jedermann et al. (2009) observaram que perdas de produtos no transporte de alimentos podem ser diminuídas por meio de uma gestão de estoques com conceito dinâmico de vida útil em vez de uma data de validade fixa ou rotulada. Os autores relatam redução de perdas de qualidade de $16 \%$ para $8 \%$ no transporte de carnes e de $15 \%$ para $5 \%$ no transporte de peixes.

Nascimento Nunes et al. (2014) analisaram a perda de qualidade em morangos, por meio do monitoramento da temperatura, desde a colheita até a distribuição para a venda no varejo. Ao compararem com uma distribuição sem o uso do monitoramento, considerando-se a ausência de informações de qualidade, concluíram que as perdas podem ser reduzidas de $37 \%$ para $23 \%$.

Jedermann et al. (2014b) relatam a aplicação de RSSF em teste-piloto, durante o transporte refrigerado de bananas em navios do Equador para a Alemanha, com transmissão de dados em tempo real, utilizando sensores de temperatura e umidade, atingindo o objetivo de redução de perda de qualidade de $5 \%$.

Tingman et al. (2010) estimaram a vida de prateleira de filé de tilápia congelada monitorando a temperatura de transporte em contêiner, por meio de RFID com sensores de temperatura. Gras (2006) analisou o monitoramento de produtos congelados usando etiquetas RFID semipassivo e ativo, com sensores de temperatura. O trabalho experimental abrangeu quatro etapas da cadeia: produção, transporte, armazenamento e entrega. Os dados coletados eram enviados para uma plataforma de gestão da cadeia de frio. Amador et al. (2009) monitoraram, por meio de RFID, a temperatura de transporte de abacaxis Crownless, a partir de um packing house da Costa Rica até o CD de uma empresa nos EUA. Os resultados mostraram que as precisões de leitura da temperatura das etiquetas RFID com sensor foram análogas às de data logger, mas apresentaram maior eficiência porque permitiram rápida leitura e melhor aquisição dos dados do ambiente refrigerado, além da possibilidade de acessar os dados coletados em qualquer ponto da cadeia de distribuição.
A cadeia logística de peixe fresco foi monitorada por Abad et al. (2009), por meio de etiqueta RFID, com sensores de luz, de temperatura e de umidade relativa. O sistema proporcionava informação em tempo real das condições de conservação do peixe, nos diferentes elos da cadeia de distribuição. A etiqueta RFID foi colocada no interior da caixa com o peixe, para garantir uma leitura mais precisa, sendo a leitura feita externamente, sem a necessidade de abertura da caixa de peixe.

Jedermann et al. (2009), em projeto-piloto, monitoraram 16 caminhões com multicompartimentos refrigerados usando RFID semipassivo, com sensores de temperatura para detectar gradientes de temperatura. Os autores concluíram que as etiquetas semipassivas podem ser usadas para monitorar a temperatura de alimentos refrigerados, identificar áreas problemáticas e antecipar a solução dos problemas com a emissão de alarmes, sendo boas ferramentas com adequado custo-benefício

Mainetti et al. (2013) utilizaram a tecnologia de radiofrequência, RFID e NFC (Near Field Communication), para a rastreabilidade e o acompanhamento de uma cadeia de abastecimento de hortaliças minimamente processadas, desde a produção até a gôndola do supermercado, nas operações de empresa de varejo italiana. A análise realizada destacou aspectos críticos da gestão da cadeia de abastecimento, definindo a reengenharia dos processos. O modelo de reengenharia foi concebido tendo em vista a exploração das potencialidades do uso combinado de tecnologia de radiofrequência, RFID e NFC, associadas às normas de padrão EPC (Eletronic Product Code).

A Rede de Supermercado Shuitema da Holanda, em 2007, iniciou o monitoramento da temperatura de hortaliças com RFID, desde a produção agrícola até a gôndola do supermercado. Com as vantagens constatadas, o projeto foi implementado para todos os fornecedores e em toda a rede de supermercado. Utilizaram etiquetas RFID padrão EPC Gen 2 UHF (SWEDBERG, 2007).

A empresa BT9, na Inglaterra, relata uma economia de 400 mil dólares após a realização de 253 transportes de cerejas em contêineres marítimos refrigerados, consequência da implantação de um sistema de monitoramento com rede de sensores, associado a um sistema de gestão FEFO (First Expire First Out) (BT9-TECH, 2015).

De acordo com Violino (2013), a empresa varejista britânica, Marks \& Spencer, aplica etiquetas RFID reutilizáveis para a rastreabilidade de alimentos frescos, em sua cadeia de suprimentos. Da mesma forma, Hy-Vee, rede de supermercados dos EUA, monitora os alimentos resfriados em sua cadeia por meio de chips RFID, com sensores de temperatura (ZAINO, 2014). Segundo Costa et al. (2013), aplicações de RFID na rastreabilidade da indústria agroalimentar são principalmente direcionadas para a cadeia da produção/distribuição, desde a produção até a sua comercialização final. 


\section{Desafios de aplicação e tendências}

Em cadeias de distribuição de alimentos, um obstáculo para a aplicação de sensores de RF (RSSF e RFID) é a resistência que determinados fatores ambientais (presença de água e metais) causam à propagação das ondas eletromagnéticas, conforme a frequência utilizada (AMADOR et al., 2009). Segundo Jedermann et al. (2014c), o alto teor de água de frutas e hortaliças impede a propagação das ondas de rádio. A distância de propagação pode ser menor do que 0,5 m (JEDERMANN et al., 2011). Mesmo sensores instalados na superfície de paletes de frutas e hortaliças podem ser afetados pelo mesmo problema, dependendo do espaço livre entre os paletes e as paredes do contêiner, sendo necessária uma análise para cada aplicação (JEDERMANN et al., 2014c). Ruiz-Garcia et al. (2010) constataram a não operabilidade de sensores localizados na parte inferior e no centro dos paletes, durante monitoramento da temperatura de alfaces, acondicionados em contêiner refrigerado de caminhão com 28 paletes em seu interior. Apenas sensores localizados na parte superior dos paletes mantiveram-se em operação. Também Kumari et al. (2015) afirmam que fatores ambientais, como poeira, umidade relativa alta, temperaturas extremas, presença de objetos metálicos e alto teor de água nos produtos, afetam o desempenho de sistemas de RFID. Um segundo desafio diz respeito a protocolos de comunicação utilizados para uma rede de sensores local e a sua compatibilidade com outras redes empregadas nos diferentes elos das cadeias logísticas. Sistemas disponíveis no mercado usam principalmente protocolos proprietários de transportes, para monitorar individualmente. Entretanto, uma cadeia de fornecimento requer um nível elevado de compatibilidade entre os sensores e dispositivos de diferentes fabricantes de sistemas de transmissão de dados (JEDERMANN et al., 2014c).

Há também esforços em andamento para unificação de normas de RFID, cujos protocolos dominantes no mercado atualmente pertencem às organizações EPC global e ISO (KUMARI et al., 2015). De acordo com Zou et al. (2014), as pesquisas em desenvolvimento de etiquetas inteligentes de RFID, ligadas em rede com multifuncionalidades, possibilitarão um grande avanço para logística inteligente de alimentos.

\section{Conclusão}

Nos Centros de Distribuição de alimentos perecíveis no Brasil, que exigem a cadeia do frio, a análise da qualidade é fundamentada em sistemas convencionais, com base em uma abordagem das características dos produtos, observadas no recebimento e sem um histórico real ou confiável de informações das condições de conservação, em toda a cadeia do frio.

No entanto, as variações das condições de conservação que, inevitavelmente, ocorrem na cadeia do frio destes produtos perecíveis alteram a realidade de sua qualidade e, consequentemente, da vida útil prevista durante a colheita ou produção, exercendo uma grande influência nas perdas de produtos.

A competitividade dos mercados globais tem exigido uma logística com prazos menores, com informações transparentes e sistemas que ofereçam segurança dos alimentos e da conservação da qualidade destes produtos frescos.

Desta maneira, a implementação de sistemas de monitoramento na cadeia do frio com RFID e RSSF, associada a modelos inteligentes de estimativa dinâmica da vida útil, apresenta um grande potencial de utilização em cadeias que já se encontram mais organizadas, inclusive possibilitando atender às crescentes exigências de rastreabilidade de produtos e de sistemas de qualidade de alimentos. Além disso, as tecnologias de RFID e RSSF têm permitido a introdução de sistemas de gestão inteligente, FEFO, substituindo o conceito atual de FIFO (First In First Out), e sendo já utilizado por cadeias de supermercados europeus na gestão de abastecimento de suas redes, trazendo maior rentabilidade. Para tanto, a utilização destas tecnologias, de forma a se tornarem rentáveis, exige análises detalhadas dos processos para que a relação custo/benefício possa ser atendida.

\section{Referências}

ABAD, E.; PALACIO, F.; NUIN, M.; GONZALEZ DE ZÁRATE, A.; JUARROS, E.; GÓMEZ, J. M.; MARCO, S. RFID smart tag for traceability and cold chain monitoring of foods: demonstration in an intercontinental fresh fish logistic chain. Journal of Food Engineering, v. 93, n. 4, p. 394-399, 2009. http://dx.doi. org/10.1016/j.jfoodeng.2009.02.004.

AMADOR, C.; EMOND, J.-P.; NUNES, M. C. Application of RFID technologies in the temperature mapping of the pineapple supply chain. Sensing and Instrumentation for Food Quality and Safety, v. 3, n. 1, p. 26-33, 2009. http://dx.doi.org/10.1007/ s11694-009-9072-6.

ASSOCIAÇÃO BRASILEIRA DE PROTEINA ANIMAL - ABPA. Relatório anual. São Paulo: ABPA, 2015. Disponível em: <http:// abpa-br.com.br/files/RelatorioAnual_UBABEF_2015_DIGITAL. pdf>. Acesso em: 20 maio 2015.

AUNG, M. M.; CHANG, Y. S. Traceability in a food supply chain: safety and quality perspectives. Food Control, v. 39, n. 1, p. 172-184, 2014. http://dx.doi.org/10.1016/j.foodcont.2013.11.007.

BADIA-MELIS, R.; MISHRA, P.; RUIZ-GARCÍA, L. Food traceability: new trends and recent advances: a review. Food Control, v. 57, p. 393-401, 2015. http://dx.doi.org/10.1016/j.foodcont.2015.05.005.

BEN-TZUR, I.; SHARSHEVSKY, H.; MANGUT-LEIBA, S.; DAGAR, $A$. The power of an integrated monitoring technology system for minimizing quality and food safety risks in fresh produce supply chain. Acta Horticulturae, v. 1079, n. 2010, p. 351-357, 2015. http://dx.doi.org/10.17660/ActaHortic.2015.1079.44. 
Monitoramento da cadeia do frio: novas tecnologias e recentes avanços

Spagnol, W. A. et al.

BRAND, D. Mode shift in perishables. [s.I.]: [s.n.], 2014. Disponível em: <http://ipaper.ipapercms.dk/MCl/Various/ SeaburyModeshiftinperishables/>. Acesso em: 20 maio 2015.

BT9-TECH. Reducing food loss: the need for a holistic approach. London: BT9-TECH, 2015. Disponível em: <http://www.bt9-tech. com/general/reducing-food-loss-the-need-for-a-holistic-approach/>. Acesso em: 20 maio 2015.

COSTA, C.; ANTONUCCI, F.; PALLOTTINO, F.; AGUZZI, J.; SARRIÁ, D.; MENESATTI, P. A review on agri-food supply chain traceability by means of RFID technology. Food and Bioprocess Technology, v. 6, n. 2, p. 353-366, 2013. http://dx.doi.org/10.1007/ s11947-012-0958-7

CUIÑAS, I.; NEWMAN, R.; TREBAR, M.; CATARINUCCI, L.; MELCON, A. A. RFID-based traceability along the food-production chain. IEEE Antennas \& Propagation Magazine, v. 56, n. 2, p. 196-207, 2014. http://dx.doi.org/10.1109/MAP.2014.6837090.

DITTMER, P.; VEIGT, M.; SCHOLZ-REITER, B.; HEIDMANN, N.; PAUL, S. The intelligent container as a part of the Internet of Things. In: 2012 IEEE INTERNATIONAL CONFERENCE ON CYBER TECHNOLOGY IN AUTOMATION, CONTROL, AND INTELLIGENT SYSTEMS (CYBER), 2012, Bangkok, Thailand. Proceedings... USA: IEEE, 2012. p. 209-214.

GAO, J. Intelligent and interactive package based on RFID and WSN. Stockholm: KTH Royal Institute Of Technology, 2011.

GARCIA, M.; BRI, D.; SENDRA, S.; LLORET, J. Practical deployments of wireless sensor networks : a survey. International Journal on Advances in Networks and Services, v. 3, n. 1, p. 170-185, 2010.

GRAS, D. RFID based monitoring the cold chain. In: INTERNATIONAL WORKSHOP COLD CHAIN MANAGEMENT, 2., 2006, Bonn. Proceedings... Bonn: [s.n.], 2006.

HAAN, G. H.; HILLEGERSBERG, J.; JONG, E.; SIKKEL, K. Adoption of wireless sensors in supply chains: a process view analysis of a pharmaceutical cold chain. Journal of Theoretical and Applied Electronic Commerce Research, v. 8, n. 2, p. 138-154, 2013.

HEIDMANN, N.; HELLWEGE, N.; PETERS-DROLSHAGEN, D.; PAUL, S.; DANNIES, A.; LANG, W. A low-power wireless UHF/LF sensor network with web-based remote supervision: implementation in the intelligent container. In: IEEE SENSORS 2013, 2013, Baltimore. Proceedings... USA: IEEE, 2013. p. 3-6.

JEDERMANN, R. Results of the project Intelligent Container. Bremen: Intelligent Container, 2014. Disponível em: <http:// www.intelligentcontainer.com/en/project-results.html>. Acesso em: 20 maio 2015.

JEDERMANN, R.; BECKER, M.; GÖRG, C.; LANG, W. Testing network protocols and signal attenuation in packed food transports. International Journal of Sensor Networks, v. 9, n. 3-4, p. 170, 2011. http://dx.doi.org/10.1504/IJSNET.2011.040238.

JEDERMANN, R.; NICOMETO, M.; UYSAL, I.; LANG, W. Reducing food losses by intelligent food logistics. Philosophical Transactions of the Royal Society A: Mathematical, Physical and Engineering Sciences, v. 372, p. 20130302, 2014a.

JEDERMANN, R.; PRAEGER, U.; GEYER, M.; LANG, W. Remote quality monitoring in the banana chain. Philosophical Transactions of the Royal Society A: Mathematical, Physical and Engineering Sciences, v. 372, n. 2017, p. 20130303, 2014b.

JEDERMANN, R.; POTSCH, T.; LLOYD, C. Communication techniques and challenges for wireless food quality monitoring. Philosophical Transactions of the Royal Society A: Mathematical, Physical and Engineering Sciences, v. 372, n. 2017, p. 2013030420130304, 2014c. PMid:24797133. http://dx.doi.org/10.1098/ rsta.2013.0304.

JEDERMANN, R.; RUIZ-GARCIA, L.; LANG, W. Spatial temperature profiling by semi-passive RFID loggers for perishable food transportation. Computers and Electronics in Agriculture, v. 65 , n. 2, p. 145-154, 2009. http://dx.doi.org/10.1016/j. compag.2008.08.006.

KADER, A. A. Postharvest biology and technology: an overview. In: KADER, A. A. (Ed.). Postharvest technology of horticultural crops. 3rd ed. Oakland: University of California Agricultural and Natural Resources, 2002. p. 39-48.

KAUSHIK, S.; SINGH, C. Monitoring and controlling in food storage system using wireless sensor networks based on zigbee \& bluetooth modules. International Journal of Multidisciplinary in Cryptology and Information Security, v. 2, n. 3, p. 7-10, 2013.

KETZENBERG, M. E.; BLOEMHOF-RUWAARD, J. M. The value of RFID technology enabled information to manage perishables. [s.l.]: [s.n.], 2009. Disponível em: <https://papers.ssrn.com/sol3/ papers.cfm?abstract_id=1376152>. Acesso em: 20 maio 2015.

KUMARI, L.; NARSAIAH, K.; GREWAL, M. K.; ANURAG, R. K. Application of RFID in agri-food sector. Trends in Food Science \& Technology, v. 43, n. 2, p. 144-161, 2015. http://dx.doi. org/10.1016/j.tifs.2015.02.005.

LANG, W.; JEDERMANN, R.; MRUGALA, D.; JABBARI, A.; KRIEG-BRÜCKNER, B.; SCHILL, K. The "Intelligent Container": a cognitive sensor network for transport management. IEEE Sensors Journal, v. 11, n. 3, p. 688-698, 2011. http://dx.doi. org/10.1109/JSEN.2010.2060480.

LÜTJEN, M.; DITTMER, P.; VEIGT, M. Quality driven distribution of intelligent containers in cold chain logistics networks. Production Engineering, v. 7, n. 2-3, p. 291-297, 2013. http:// dx.doi.org/10.1007/s11740-012-0433-3.

MAINETTI, L.; MELE, F.; PATRONO, L.; SIMONE, F.; STEFANIZZI, L. M.; VERGALLO, R. An RFID-based tracing and tracking system for the fresh vegetables supply chain. International Journal of Antennas and Propagation, v. 2013, p. 1-15, 2013. http:// dx.doi.org/10.1155/2013/531364

MEJJAOULI, S.; BABICEANU, R. F. RFID-wireless sensor networks integration: decision models and optimization of logistics systems 
Monitoramento da cadeia do frio: novas tecnologias e recentes avanços

Spagnol, W. A. et al.

operations. Journal of Manufacturing Systems, v. 35, p. 234-245, 2015. http://dx.doi.org/10.1016/j.jmsy.2015.02.005.

NASCIMENTO NUNES, M. C.; NICOMETO, M.; EMOND, J. P.; MELIS, R. B.; UYSAL, I. Improvement in fresh fruit and vegetable logistics quality: berry logistics field studies. Philosophical Transactions of the Royal Society A: Mathematical, Physical and Engineering Sciences, v. 372, n. 2017, p. $20130307-$ 20130307, 2014.

OGASAWARA, A.; YAMASAKI, K. RFID-based solutions: a temperature-managed traceability system using RFID tags with embedded temperature sensors. NEC Technical Journal, v. 1, n. 2, p. 82-86, 2006.

PEDROSO, M. C.; ZWICKER, R.; DE SOUZA, C. A. Adoção de RFID no Brasil: um estudo exploratório. Revista de Administração, v. 10, n. 1, p. 12-36, 2009.

RIZZO, F.; BARBONI, M.; FAGGION, L.; AZZALIN, G.; SIRONI, $M$. Improved security for commercial container transports using an innovative active RFID system. Journal of Network and Computer Applications, v. 34, n. 3, p. 846-852, 2011. http:// dx.doi.org/10.1016/j.jnca.2010.04.006.

RUIZ-GARCIA, L. Development of monitoring applications for refrigerated perishable goods transportation. Madrid: Universidad Politécnica de Madrid, 2008.

RUIZ-GARCIA, L.; LUNADEI, L. Monitoring cold chain logistics by means of RFID. In: TURCU, C. (Ed.). Sustainable radio frequency identification solutions. Vukovar: Intech, 2010. p. 37-50. http://dx.doi.org/10.5772/8006.

RUIZ-GARCIA, L.; LUNADEI, L. The role of RFID in agriculture: applications, limitations and challenges. Computers and Electronics in Agriculture, v. 79, n. 1, p. 42-50, 2011. http:// dx.doi.org/10.1016/j.compag.2011.08.010.

RUIZ-GARCIA, L.; LUNADEI, L.; BARREIRO, P.; ROBLA, J. I. $A$ review of wireless sensor technologies and applications in agriculture and food industry: state of the art and current trends.
Sensors, v. 9, n. 6, p. 4728-4750, 2009. PMid:22408551. http:// dx.doi.org/10.3390/s90604728.

RUIZ-GARCIA, L.; STEINBERGER, G.; ROTHMUND, M. A model and prototype implementation for tracking and tracing agricultural batch products along the food chain. Food Control, v. 21, n. 2, p. 112-121, 2010. http://dx.doi.org/10.1016/j.foodcont.2008.12.003.

SHAN, Q.; LIU, Y.; PROSSER, G.; BROWN, D. J. Wireless intelligent sensor networks for refrigerated vehicle. In: CIRCUITS AND SYSTEMS SYMPOSIUM ON EMERGING TECHNOLOGIES: FRONTIERS OF MOBILE AND WIRELESS COMMUNICATION, 6., 2004, Shanghai. Proceedings... Shanghai: IEEE, 2004. Disponível em: <http://ieeexplore.ieee.org/lpdocs/epic03/wrapper. htm?arnumber=1321941>. Acesso em: 20 maio 2015.

SWEDBERG, C. Schuitema ponders future of fresh-chain pilot. RFID Journal, Hauppauge, 10 dez. 2007. Disponível em: <http://www. rfidjournal.com/articles/view?3793>. Acesso em: 10 ago. 2014.

TINGMAN, W.; JIAN, Z.; XIAOSHUAN, Z. Fish product quality evaluation based on temperature monitoring in cold chain. African Journal of Biotechnology, v. 9, n. 37, p. 6146-6151, 2010.

VIOLINO, B. Marks \& spencer rolls out RFID to all its stores. RFID Journal, Hauppauge, mar. 2013.

WHITE, J. How cold was it? Know the whole story. Food Logistics, [s.l.], set. 2007, p. 38-40.

ZAINO, J. Hy-vee supermarkets track perishables to ensure freshness. RFID Journal, Hauppauge, jul. 2014.

ZHANG, M.; LI, P. RFID application strategy in agri-food supply chain based on safety and benefit analysis. Physics Procedia, V. 25, p. 636-642, 2012. http://dx.doi.org/10.1016/j.phpro.2012.03.137.

ZOU, Z.; CHEN, Q.; UYSAL, I.; ZHENG, L. RFID enabled wireless sensing for intelligent food logistics. Philosophical Transactions of the Royal Society A, v. 372, n. 2017, p. 20130313, 2014. http://dx.doi.org/10.1098/rsta.2013.0313. 